\title{
Circulations et occupations préhistoriques et protohistoriques dans la vallée du Furon
}

$n^{\circ} 2211192$

Jocelyn Robbe

\section{(2) OpenEdition \\ Journals}

Édition électronique

URL : http://journals.openedition.org/adlfi/15190

ISSN : 2114-0502

Éditeur

Ministère de la culture

\section{Référence électronique}

Jocelyn Robbe, «Circulations et occupations préhistoriques et protohistoriques dans la vallée du

Furon », ADLFI. Archéologie de la France - Informations [En ligne], Rhône-Alpes, mis en ligne le 30 juillet 2015, consulté le 30 avril 2019. URL : http://journals.openedition.org/adlfi/15190

Ce document a été généré automatiquement le 30 avril 2019

(c) Ministère de la Culture et de la Communication, CNRS 


\section{Circulations et occupations préhistoriques et protohistoriques dans la vallée du Furon}

$n^{\circ} 2211192$

Jocelyn Robbe

Code INSEE de la commune : $38153,38205,38433,38474$

Lien Atlas (MCC) :

http://atlas.patrimoines.culture.fr/atlas/trunk/index.php?

ap_theme=DOM_2.01.02\&ap_bbox=5.583;45.151;5.652;45.235

\section{Campagne 2013}

1 La dernière campagne de prospection de la vallée du Furon a eu lieu du 6 août au 17 août 2013. Pour mémoire, rappelons que cette zone de prospection est étagée entre $200 \mathrm{~m}$ et $1500 \mathrm{~m}$ d'altitude et couvre environ $31 \mathrm{~km}^{2}$. La D531 qui relie Sassenage à Lansen-Vercors serpente au fond de cette vallée. Nous avons divisé cette zone en trois secteurs. Le premier se développe entre le village de Lans-en-Vercors (alt. $1000 \mathrm{~m}$ ) et celui d'Engins (alt. $940 \mathrm{~m}$ ) et couvre environ $14 \mathrm{~km}^{2}$. Le deuxième s'étend du village d'Engins au site de la Grande Rivoire (alt. $580 \mathrm{~m}$ ) et représente également une surface d'environ $14 \mathrm{~km}$ ${ }^{2}$. Enfin, le troisième secteur correspond à la zone située entre le site archéologique de la Grande Rivoire et la ville de Sassenage (alt. $210 \mathrm{~m}$ ) et couvre environ $3 \mathrm{~km}^{2}$. Sa superficie plus réduite nous a permis de mener la campagne en 15 jours à deux personnes.

Cette dernière portion de la vallée est très urbanisée et une partie est occupée par les carrières Vicat et Balthazard \& Cotte. En outre, ce secteur a été recouvert par au moins quatre épisodes d'écroulements, provenant de la Dent du Loup qui le surplombe (Gamond et al., 2008). 
3 Nous avons tout d'abord mené une prospection classique dans le peu de parcelles cultivées restantes. Nous n'avons rencontré aucun vestige archéologique (pré- ou protohistorique). Il nous a ensuite fallu mener une enquête de proximité auprès des habitants et des industriels. Ainsi, nous avons contacté l'association «Les côtes de Sassenage ", qui a aimablement diffusé un mail à ses adhérents. Nous leur avons demandé de bien vouloir nous signaler toutes découvertes de vestiges qu'ils auraient pu faire lors de leurs travaux de jardinages ou d'aménagements. M. Vartanian a accepté de nous recevoir dans sa propriété du Château de Beaurevoir. Le parc qui date du XIX ${ }^{\mathrm{e}}$ s., occupe 30 ha du hameau des Côtes de Sassenage. Il surplombe une partie du torrent du Furon et comporte à l'est, un piton rocheux où se trouvent les ruines d'un ancien château datant $\mathrm{du} \mathrm{XII}^{\mathrm{e}} \mathrm{s}$. C'est donc un lieu intéressant d'un point de vue topographique, et qui a pu motiver des occupations pré ou protohistoriques. En revanche, les possibilités d'en trouver des preuves restent faibles en regard des importants aménagements médiévaux établis sur la zone.

Nous avons aussi pris contact avec la carrière Vicat qui exploite une partie du calcaire marneux sur le plateau des Côtes de Sassenage. M. Fayolle, le responsable, a proposé de nous fournir des documents en rapport avec cette exploitation. Cependant, nous n'avons pas eu l'autorisation de visiter la carrière.

M. Pichon directeur de la carrière Balthazard \& Cotte nous en a spontanément ouvert les portes. Celle-ci exploite les flancs des falaises sénoniennes au pied des Côtes de Sassenage. À la suite d'un long entretien avec M. Pichon, géologue de formation, il est apparu difficile de découvrir des vestiges préhistoriques ou protohistoriques dans ce secteur fortement remanié. En effet, avant l'exploitation du plateau par les carriers, celui-ci était recouvert de vergers et de vignes au début du $\mathrm{XIX}^{\mathrm{e}} \mathrm{s}$. Par la suite, des batteries militaires furent construites et enfin de nombreuses petites carrières furent exploitées dès la fin du $\mathrm{XIX}^{\mathrm{e}} \mathrm{s}$. De plus, l'écroulement de la Dent du Loup, daté pour le secteur des carrières de 6000 ans BP grâce à des concrétions calcaires trouvées dans la carrière Vicat, laisse peu d'espoir de mettre au jour des vestiges.

6 De nombreux blocs métriques à décamétriques encore visibles dans les sous-bois surplombant les carrières témoignent de cet écroulement.

7 Une partie de la campagne a également été consacrée à la circulation au sein de la vallée afin de replacer le site de la Grande Rivoire dans son contexte.

8 Ainsi, dans l'optique de déplacements de bergers néolithiques accompagnés d'animaux domestiques (chèvres ou moutons), deux principaux axes se dessinent après avoir été directement testés sur le terrain :

- L'un passe par les gorges du Furon en longeant au plus près le torrent. Les zones de canyon peuvent être évitées en empruntant des sentiers les surplombant.

- Le second axe possible chemine en crête, mais implique un itinéraire de plus longue durée pour accéder aux plateaux. Quoi qu'il en soit, à partir de la Grande Rivoire, l'accès aux gites de silex (pointés lors de la campagne 2012) à l'est ou aux éventuelles zones de pâturages, se fait en moins de deux heures.

9 Il est à noter que les déplacements en pieds de falaises, qui offrent une bonne ligne directrice dans un environnement forestier dense, restent possibles pour un groupe de chasseurs mais qu'ils sont plus délicats pour des bergers avec un troupeau.

Ces axes de circulations se rejoignent tous au village d'Engins qui fait figure de carrefour renvoyant aux plateaux adjacents. 
11 Le hameau des Côtes de Sassenage occupe une place privilégiée au sein de la vallée du Furon. Il offre un point de vue non négligeable sur la vallée du Drac en contrebas, et constitue une zone de replat avant de s'engager dans les gorges du Furon et d'atteindre les plateaux. Malheureusement, la forte urbanisation et l'activité industrielle de ce hameau ainsi que les différents épisodes d'écroulement de la Dent du Loup nous laissent peu d'espoir de retrouver des vestiges archéologiques correspondant aux périodes préhistoriques et protohistoriques. Nous espérons malgré tout que les démarches entreprises auprès des habitants et des exploitants nous apporteront des éléments de réponse sur la fréquentation pré- et protohistorique de ce plateau de piémont. La campagne de cette année nous a aussi permis d'avoir une idée plus précise de la circulation au sein de la vallée du Furon.

\section{BIBLIOGRAPHIE}

Gamond et al., 2008 : Gamond : Datation et chronologie relative des épisodes d'écroulements sur le versant est de la Dent du Loup et implication pour l'estimation du risque, LGIT, Observatoire de Grenoble, EDYTEM université de Savoie, 2008.

\section{INDEX}

Index géographique : Rhône-Alpes, Isère (38), Engins, Lans-en-Vercors, Saint-Nizier-duMoucherotte, Sassenage

operation Prospection thématique (PRT)

\section{AUTEURS}

\section{JOCELYN ROBBE}

Chercheur bénévole 